\title{
Addressing the Crisis in Campus Counseling Centers: An Organizational Perspective
}

\section{Tavallali LM* and Cox RW}

University of Akron, USA

*Corresponding author: Lisa M Tavallali, University of Akron, Lake Erie College of Osteopathic Medicine 4800 Lakewood Ranch Blvd, Bradenton, FL 34211, USA, Tel: 941-405-1558; Email: ltavallali@lecom.edu

\section{Review Article}

Volume 2 Issue 5

Received Date: July 02, 2019

Published Date: September 13, 2019

DOI: $10.23880 /$ jqhe- 16000136

\section{Abstract}

The challenges for those responsible for the mental health of students on the campus are multi-faceted and complex. These medical challenges are exacerbated by the administrative realities that there is no consistent process for seeking a standard of mental health care on college campuses. This is not about the professionalism of those in counseling centers. This preliminary study examines the organizational processes and practices that are used to help students. Two topics stand out from this analysis. First is that the needs of the student/client are too complex for the simplistic self-reporting entry systems. Second is that the current models fail to capture critical and crisis needs and may not even be effective at providing timely assistance for the least complex and most straight-forward, much less the more critical problems.

Keywords: Mental Health Counseling Practices; Organizational Structure; Continuum of Care

Abbreviations: ACHA: American College Health Association's; ADA: Americans with Disabilities Act; AUCCCD: Association for University and College Counseling Center Directors CCAPS: Counseling Center Assessment of Psychological Symptoms.

\section{Introduction}

A century ago the first counseling and mental health program was introduced at Princeton in 1910 [1]. Often little more than an extension of the "infirmary" by the 1960s, counseling programs was common. Much has changed for students since the $1960 \mathrm{~s}$, much less the beginning of the 20th century. No longer is "going to college" the preserve of the young and wealthy. College campuses are rarely the tight-knit, homogeneous villages of the select. No longer are they places for a few who share social experiences (students are still in or barely beyond their teens), gender and economic status. Many campuses today are virtually mid-sized cities with diverse and large populations. Public universities not uncommonly have tens of thousands of students. The challenges for those responsible for the mental health of students on the campus are multi-faceted and complex.

Even as students enroll at an ever-increasing rate, the number of college counselors available to assist these students with mental health issues is not expanding at the same level [2]. There are times when students are in crisis mode and are turned away from college counseling centers at critical times due to no available staff to help. Many students are then referred to outside community mental health centers, but these organizations are also at maximum capacity which can leave the student in a dangerous situation. Thus, these high rates of mental health and limited rates of treatment utilization present 


\section{Journal of Quality in Health Care \& Economics}

significant concerns for campus administrators, parents, and other stakeholders [3].

In the last two decades higher education counseling departments' patients have expanded not only in size but in complexity of issues as well, by shifting from developmental and informational concerns to more severe psychological problems. In addition, students who might never have been able to attend college before due to behavioral issues or mental illness are now able to attend because of improved treatment options [4]. Of particular concern are those students contemplating suicide. Suicide death among college students is a highprofile topic, as suicide ranks second as the leading cause of death among college students [5].

These medical challenges are exacerbated by the administrative realities that there is no consistent process for seeking a standard of mental health care on college campuses. Not surprisingly the decision models are shaped by budgetary and personnel concerns [6]. The adage of "doing more with less" is as pertinent on a college campus as anywhere. This preliminary study examines the organizational processes and practices that are used to help students. As will be laid out below, the organizational practices of most counseling centers are based upon assumptions and decision-making models that go back to the very earliest counseling programs. Thus, the starting point for this examination is a review of current policies followed up with the administrative and institution barriers to effective delivery of mental health services on college campuses.

Current Policies for Mental Health Services for College and University Students

To protect both the student and the school, administrative protocol and policy must be considered to address concerns for both parties. Under the parameters of the Americans with Disabilities Act (ADA), "which prohibits discrimination against students whose mental problems "substantially limit a major life activity," including learning, colleges and universities are required to provide "reasonable accommodations" to protected students which include reasonable modifications to normal rules and procedures to allow those students to continue and succeed in higher education" [7].

Although the demand for comprehensive mental health services continues to increase, many university budgets remain unchanged or have increased only slightly from past years according to the 2013-2014 Association for University and College Counseling Center Directors (AUCCCD) survey [8]. AUCCCD survey data suggest that larger schools have struggled to reach pre-2008 recession budget levels, reflecting in fewer counseling clinicians to student ratios. The current recommended student to counselor ratio is 1 to $1,000-1,500$ to meet the needs of the demand of students at colleges across the country, depending on services offered and other campus mental health agencies. The vulnerability increases as the center's ratio increases [9]. This ratio was established using a combination of empirical analysis and judgment of experienced directors who were leading experts in their field.

\section{Barriers to Service for Students}

Many people with mental health conditions who could benefit from available treatments do not receive the care available to them due to several barriers generally understood to be limited knowledge, inadequate health insurance coverage, and stigma [10]. Sophisticated approaches are needed to help reduce or eliminate these and other barriers. College counseling centers across the country play a major role in this issue as they continue to report an increase in clients with severe psychological problems year over year as reported to the Association for University and College Counseling Center Directors [11]. By reducing barriers in college student populations, timely and effective treatment may offer substantial longterm benefits for the remainder of their lives.

\section{Organizational Factors}

Based upon the historical record, the organizational framework for counseling centers can be characterized as a simple input-output model. Into the 1960s applicants for college admissions with mental health issues would been denied admission, so accepting the students as a blank slate at admission was not surprising. At the other end of the process (output) would be a student who was helped through the process and either leaves or graduates.

\section{Design of the Study}

The question that must be asked is whether the logic of the organizational processes is part of the problem. Only by a more careful examination of the organizational processes and exploring whether an alternative organizational process model might better serve students can centers get past a cycle of a focus on inputs.

Data to be analyzed in this study comes from multiple sources. Secondary sources are from the results of the 


\section{Journal of Quality in Health Care \& Economics}

most recent reports gathered by the Association for University and College Counseling Center Directors, American College Health Association, and Center for Collegiate Mental Health.

To supplement these secondary sources a questionnaire was developed to gather data on the following topics:

1. What is the process for students presenting for services to the counseling center?

2. What is the primary barrier to services that students say is their reason for the lack of ability to obtain services at these institutions?

3. Do counseling centers tend to have a wait list, and if so, what is the average length of wait time coutside of initial intake)?

4. If there has been a change in the amount of resources provided to their center, how have counseling centers adjusted their processes to accommodate the needs of students?

5. If these centers have experienced an increased demand, in volume and/or complexity, how have they managed these challenges?

The questionnaire went through a series of changes, at each stage being evaluated by several counselors to strengthen its validity. To gain insight into the workings of counseling centers, a pilot study for the questionnaire was developed and appointments were made with a select number of counseling center directors. Several meetings took place for counselors to share their recommendations about the preliminary questionnaire and to learn more about the workings of college counseling centers in general. The final version consisted of eight questions and is displayed in Appendix 1.

Appendix 1: Survey Used for Study.

Questions for Mental Health discussion on college campuses

1. Is the school you are reporting on

a) Public

b) Private

2. What is the intake process used for students who walk in requesting counseling?

a) Schedule an appointment for them

b) Have them seen by someone at that time

c) Have them complete paperwork and tell them someone will contact them

d) Phone triage

e) Other

3. Do you have a wait list for students to be seen by a mental health provider (not including intake)? If so, what is the average length of time?

a) Yes No b) 24-48 hours 3-5 days 5-7 days $7-10$ days $>10$ days

4. What services, if any, do you have to send out to a community provider?

What are your decision rules for determination?

5. Do you think students are presenting with more complex problems than the past 10 to 15 years? If so, why?

6. What is the primary barrier to services for students at your institution, if any?

a) Stigma

b) Access due to limited resources

c) Cost

d) Do not have appropriate staff for diagnosis

e) Want to figure it out themselves

7. If you have experienced a change in the amount of resources (funding, staffing, etc.), how have you adjusted your processes to still accommodate the needs of students?

8. If you have experienced increased demand, in volume and complexity, of students in college today, how have you managed these challenges?

a) Offered group sessions

b) Utilization of other modalities (online programs)

c) Refer to outside provider

d) Limit number of sessions for others to be seen

Importantly, the interviews which helped refine the questionnaire served as an important source of "stories" and depth of detail. In effect this questionnaire development process served to create a fourth data source.

\section{Population}

The population for the survey study consisted of 213 counseling directors at both public and private colleges and universities having enrollment greater than 10,000. For this study institutional size is an important measure because it relates to institutional structure, campus culture, and other factors that could influence psychosocial outcomes [12]. Using the Carnegie Foundation's classification of institutional size, it was determined that only schools classified as large (greater than 10,000) were included [13]. While it was important to include both public and private schools in the distribution of the questionnaire, the use of a "large university" basis for the survey meant that there are far more public universities than private in the population. The size of staff and resources ultimately was more important than the possible public-private differences. Each counseling director was contacted by email, phone and/or direct interview to obtain information for the 


\section{Journal of Quality in Health Care \& Economics}

study through a questionnaire developed to obtain additional information not readily found in the literature.

\section{Findings}

Who Works in the Centers?

There are a variety of types of professional providers at counseling centers throughout the country. The following is a compilation of the primary professional counselors which provide services to students in order of majority: clinical psychologists, counseling psychologist, professional counselor, social worker, psychiatrist, marriage and family therapist, other mental health professional, and psychiatric nurse practitioner [14].

\section{Center Operations}

An important factor to consider is the number of months counseling centers provide services to students. Slightly less than $80 \%$ do so. $16 \%$ are open only $8-9$ months a year (essentially the traditional academic year). In addition, the same survey states that greater than $52 \%$ of the schools report that their counseling center offers services outside the normal $8 \mathrm{am}-5 \mathrm{pm}$ daily business hours of operation.

The 2016 AUCCCD Study found that over 20\% of counseling centers had their operating budget decreased over the past year, while 55\% remained the same and $23 \%$ achieved a slight increase. The net change in FTEs (Full Time Equivalent) in the past year for the 529 centers responding to the survey revealed a 403.57 FTE increase in staffing with the majority being professional/clinical (259.69). This is reflective of the increased demand that schools are facing each semester. One hundred and eighty-six schools or 36\% reported having a waitlist for students to receive ongoing treatment while at the same time more than $54 \%$ of the schools do limit the number of counseling sessions allowed by students.

\section{Treatment Protocols}

Data gathered from counselors regarding termination of counseling for students was reported for the first time in the CCMH 2016 report. The data was gathered utilizing the Counseling Center Assessment of Psychological Symptoms (CCAPS) form which is a multidimensional assessment and outcome-monitoring tool used by $\mathrm{CCMH}$ counseling centers and most other college and university counseling centers. Three findings of note are:

1. "Client drop-out" plays a role in one-third of all terminations.
2. Institutional policies (such as session limits or fees) plays an important role in determining how treatment ends

3. Treatment concerns at termination mirror presenting concerns at the start of treatment

The average number of counseling sessions for a student is 4.66 , but this number is inflated with a relative small number of students utilizing much of the services. Ten percent of students accounted for $37 \%$ of services provided. In contrast, the most common number of appointments was only one.

The American College Health Association's (ACHA) spring 2016 report consisted of 137 participating schools with 95,761 students responding $(65.6 \%$ female, $31.3 \%$ male, non-binary $3.1 \%$ ). While $84.3 \%$ of students described their overall general health as either, good, very good, or excellent, $36.7 \%$ said they felt so depressed that is was difficult to function. Almost 9,400 (9.8\%) students seriously considered suicide and approximately 1,435 $(1.5 \%)$ of these students attempted suicide at some point in their life. Over the 12 months of the study, $54.7 \%$ of students reported that they had experienced "more than average stress" or "tremendous stress".

\section{Question Response Summary}

While many counseling centers on college campuses provide similar services to students at their centers, each is challenged in its own way and has worked to develop modifications to increase efficiencies to better serve those who are not getting into the system. While not all students are triaged when they first seek services, it appears that the majority of counseling centers do some type of initial meeting (68\%), regardless of brevity, to determine if the student is in crisis and needs attention immediately.

In the general population, one of the primary barriers for most individuals not to receive care has been found to be the stigma attached to mental health illness [15]. The primary reason that students do not receive mental health services appears to be lack of access due to limited resources (57\%). For example, although most schools do not have a wait list (70\%), those who did have a wait list (30\%) reported an average delay of 2 weeks with some extending to 4 weeks. Although many university counseling center directors report they do not have a wait list for scheduling students to be seen per se, students seeking help are being scheduled out 1 to 5 weeks for an appointment. The controversy here is "how long is too long to wait to be seen?" and what critical issues could be missed with these delayed interventions. In addition to a 


\section{Journal of Quality in Health Care \& Economics}

delay in getting an appointment, the care that is provided tends to be episodic rather than ongoing care while most of these students require care over a greater period. Furthermore, those schools that did not have access for students at any time ended up turning them away and offered to refer them to a community resource.

There are mixed responses regarding resource allocation adjustments as well as complexity level of students presenting to counseling centers today, but directors and staff have developed many alternatives to continue to provide care to the vast number of students who are seeking care from them. By utilizing group sessions (85\%), workshops, and other modalities of care such as web-based app programs (34\%), more students requesting services can be offered additional options in addition to the traditional individual counseling session. Incorporating a stepped and/or integrated model of care, short term model of care, and session limits (45\%), counseling centers allow more access for more students who may not have been able to be seen due to lack of capacity.

While demand outpaces supply regarding students presenting with mental illness on college campuses today, those who are responsible for making policy and budgeting decisions must address the barriers associated with receiving mental health treatment that are within their bounds. Several contributing factors, such as increased access for more complex students now able to attend college, and a misconception of no wait list for students seeking help, when in fact the schedule is booked out 2 to 4 weeks, are causing major concerns for many stakeholders.

\section{Discussion, Implications, Recommendations}

Many changes have evolved in college and university counseling centers over the last several decades. The increased number of students struggling with mental and behavioral health problems seeking treatment is bringing attention to an alarming trend. A change in the accommodations of students presenting to colleges and universities appears to have shifted from developmental and informational needs, such as balancing social and academic demands, to more psychological in nature, such as depression and anxiety. Historically, college counseling centers were conceptualized as settings where students could be helped through a specific developmental challenge or adjustment problem such as relationship issues or homesickness, but now college counseling centers find themselves providing services for larger numbers of more seriously troubled students over longer periods of time.

\section{Implications}

For many decades college counseling centers have played a large role in providing behavioral and mental health services for students in higher education. Recognizing the increased demand for counseling centers to address the mental health needs of those students seeking out help is crucial to all stakeholders; but those are just the students who acknowledge they are in crisis. There are other students who may be unaware of the resources available to them or choose not to seek help for numerous reasons. Still other students may not be sure how to respond when they do reach out to a counseling center and are asked if this is an emergency. They may have doubts regarding their feelings or level of distress and are unsure how to reply.

Key issues that emerged include:

- The need for directors, other counseling staff, and stakeholders to be aware of the prevailing shift occurring with students on college campuses.

- To be cognizant of the recent spike in more acute cases and realize that perhaps more treatment than counseling is now being provided.

- The need to continue to adjust services as needed to serve those students asking for help, especially those with escalating thoughts of harm to self or others, should be of utmost importance.

- Discussion among stakeholders to determine if the current model of care being used is effective and most beneficial for students being provided mental health treatment today.

- Although the present delivery care model has been used in institutions of higher learning over the past one hundred years, the students seeking mental health services today are not the same as those from 100 years ago.

After such a long time and especially because of the changes of the last two decades, it must be asked if this is still the best model of care to continue using or should we look to develop a new care system which would better serve the new and different generation of students presenting to college counseling centers of today.

A practice implication identified concerns counselors recognizing what appears to be a shift of students presenting with more complex issues than seen in the 


\section{Journal of Quality in Health Care \& Economics}

past 10 to 15 years and may be the result of an influx of students who may not have been able to attend college previously. Young people who in the past were unable to attend college due to mental and behavioral issues, such as autism spectrum disorders or ADHD, are now receiving improved treatment approaches and are better controlled with medication and therapy. But many still have social skill deficits that are problematic and require special accommodations to prepare them for the academic challenges of higher education and to promote learning and retention. While counselors are not getting paid based on volume of students seen, they are triaging them and addressing the current need of the moment rather than providing them tools to develop resilience to cope with stress and adversity better.

Another implication is realizing that tackling the mental health crisis on college campuses cannot fall solely on the shoulders of counseling centers. The entire campus community must commit and get involved in this initiative. Collaboration and dedication from a large cluster of campus community stakeholders, including faculty, college administrators and student services personnel, can facilitate adequate resources that can result in more aggressive intervention and increased awareness for these students seeking mental health services. Research shows that students who participate in counseling services have reported an increase in student persistence and retention as well as improvements in their satisfaction with the quality of life.

Another practice implication relates to barriers to service for students requesting mental health counseling. According to Eisenberg et al, "Stigma associated with mental illness has been identified as a key attitudinal factor that may impede mental health service use" for the general population [16]. Students often feel uncomfortable navigating treatment for a severely stigmatized issue such as depression or anxiety. While self-disclosure was one of the reported barriers to service for students, this researcher found that more than $50 \%$ of center directors reported a lack of access due to limited resources was the primary barrier for students who were not receiving mental health care on college campuses.

Finally, Herbert Simon's decision theory, provides insight into who the appropriate personnel are who should be included to work in the college counseling center [17]. For Simon, his focus of the theory is an analysis of how decisions are made and how they might be made more effectively by analyzing the decision process piece by piece and attempting to determine what then happens as each individual decision is made. Based on this theory and assessing the current resources available, counseling directors may consider a different staffing model and make changes to promote a culture of wellness and prevention to serve students before their situation escalates to a crisis.

\section{Recommendations}

The current college counseling mental health system is broken or at the very least is so overburdened that it is collapsing. The prevailing method of delivering mental health services using an input-driven approach (a student presenting to the counseling center in a distressed state and a counselor attempting to deescalate the situation, without much follow up involved), needs to be reviewed and updated to address the changing shift occurring in both demand and complexity of students' mental health needs on current college campuses. Administering triage for each crisis as it presents itself will not enable emotional stability, hence the inability to deliver improved learning outcomes. By ignoring the problem and putting a temporary bandage on it only addresses the issue for the student during the crisis point and will not create sustainability for students or the system moving forward. If we continue to do the same thing and expect a different result, we are fooling ourselves. This researcher would like to make several recommendations for further consideration.

The first onset of lifetime mental disorders normally occurs by age 24 in $75 \%$ of cases [18]. By reducing barriers in college student populations and decreasing negative attitudes surrounding mental illness within the campus community, timely and effective treatment may offer substantial long-term benefits for the remainder of their lives.

Perhaps we should consider evaluating the movement currently taking place in primary healthcare which is utilizing a model of wellness, prevention and coordination of care rather than that of being reactive and performing triage once a situation has escalated to a crisis? As traditional healthcare moves to preventative medicine or well care, instead of sick care, it has become more costeffective to care for patients while improving their overall well-being.

By attempting to anticipate potential problems and address them proactively rather than waiting for them to occur and then attempt to recover from a crisis, more progressive healthcare providers are foregoing the 


\section{Journal of Quality in Health Care \& Economics}

traditional fee-for-service care model (getting paid by the number of services/visits provided, instead of holistically treating the patient) and focusing more on value-based care (financial incentive for lowering cost and improving the quality of care for patients). Rather than treating the heart attack, the focus has switched to treating the high cholesterol to prevent the heart attack. It would behoove the mental health community to begin analyzing these same concepts.

Emphasis must be placed on the importance of students understanding that for many, the behavioral or mental health condition they are being treated for does not go away when they leave one place and go to another. Students must learn that treatment is to be continuous to remain mentally healthy. Again, as we look to the recent mainstream physical healthcare model of delivery, we see that the need for continuity of care is a critical component for the model to be successful. According to the Healthcare Information and Management Systems Society, continuum of care "is a concept involving a system that guides and tracks patients over time through a comprehensive array of health services spanning all levels and intensity of care. The Continuum of Care covers the delivery of healthcare over a period of time" (www.himss.org/definition-continuum-care). By utilizing this concept and involving an integrated system for students struggling with mental health issues to make certain there is no lapse in care, the physical and mental health delivery care team can communicate with each other and track the patient over time to ensure the student receives continued care when indicated, and monitor for intervention to prevent relapse and recurrence, as well as provide after-care.

We can also look to develop a different path for entry into the maze of receiving both physical and mental healthcare by encouraging students to seek help first with their primary care provider. By strengthening the knowledge and resources of primary care providers to recognize, respond effectively and/or refer students with more acute needs to a mental or behavioral health specialist, perhaps we can create a more connected culture so students don't get lost in the system. Utilizing a care coordinator or case manager can assist in helping to navigate the current complicated healthcare delivery system and attempt to prevent discontinuation of necessary medication or follow up care leading to emotional escalation or crisis.

By using a decision theory model, the critical steps that are taken as students enter the mental health counseling centers on college campuses, or even prior to enrollment, can be identified and the processes that determine next steps can be established based on the factors presented by students at intake or through care management. Utilizing a decision tree to represent these channels will also allow for clarity as to proper assignment of level and type of counselor for each student. This design may also provide insight into barriers to service and processing wait lists for students delayed in being seen.

In changing the current delivery model and engaging in a more active, strategic approach, schools can better anticipate and evaluate clinical and programming needs. The college experience is not isolated to the classroom alone; stakeholders are called upon to reflect on their priorities and examine the needs of a diverse student population. Implementing new alternatives, such as a crisis hotline, may offer an opportunity to delay or prevent a tragedy due to inadequate access and/or resources for students in need.

Counseling staff should also look for opportunities to engage students outside the counseling center, especially first year students, by promoting overall health and helping students identify mental health concerns in themselves and others. Overall campus engagement provides an understanding of the necessity of having behavioral and mental health services available on campus and how to assist students in accessing these services if necessary. This engagement can allow for awareness of mental health in the hope that conversation will ensue and stigma may decrease.

\section{Conclusion}

Two topics stand out from this analysis. First is that the needs of the student/client are too complex for the simplistic self-reporting entry system that worked for several decades but 20-30 years ago became unrealistic. The stigma of mental health challenges remains. Expecting to effectively serve students with walk-in and self-reporting controls costs by under-representing the problem but provides no realistic data on true demand (and true costs). Second is that simple decision models and decision-tree criteria should be used to assess students at entry. The current models fail to capture critical and crisis needs and may not even be effective at providing timely assistance for the least complex and most straight-forward, much less the more critical problems. 


\section{Journal of Quality in Health Care \& Economics}

\section{References}

1. Prescott HM (2007) Student bodies-the influence of student health services in American society and medicine. Ann Arbor University of Michigan Press.

2. Center for Collegiate Mental Health (2016) 2015 Annual Report (Publication No. STA 15-108).

3. Lipson SK, Gaddis SM, Heinze J, Beck K, Eisenberg D (2015) Variations in Student Mental Health and Treatment Utilization Across US Colleges and Universities. Journal of American College Health 63(6): 388-396.

4. Blom S, Beckley S (2005) 6 major challenges facing student health programs. The Chronicle of Higher Education.

5. Potter L, Silverman M, Connorton E, Bradshaw M (2004) Suicide Prevention Resource Center: Promoting Mental Health and Preventing Suicide in College and University Settings (Department of Health and Human Services, Substance Abuse and Mental Health Services Administration). MA: Education Development Center, Newton.

6. Heppner P, Neal G (1983) Holding up the mirror: research on the roles and functions of counseling centers in higher education. The Counseling Psychologist 11(1): 81-98.

7. Bazelon Center for Mental Health Law (2007) Supporting Students: A Model Policy for Colleges and Universities.

8. Reetz DR, Krylowicz B, Mistler B (2013) The Association for University and College Counseling
Center Directors Annual Survey. Indianapolis, Indiana.

9. International Association of Counseling Services Inc (2017) Statement Regarding Recommended Staff to Student Ratios.

10. Mechanic D (2002) Removing Barriers to Care Among Persons With Psychiatric Symptoms. Health Affairs 21(3): 137-147.

11. Carnegie Foundation for the advancement of teaching (2017) Carnegie Classifications [Fact sheet].

12. Corrigan PW, Druss BG, Perlick DA (2014) The Impact of Mental Illness Stigma on Seeking and Participating in Mental Health Care. Psychological Science in the Public Interest 15(2): 37-70.

13. Reetz DR, BershadC, LeViness P, Whitlock M (2016) The Association for University and College Counseling Center Directors. Annual Survey.

14. Eisenberg D, Downs M, Golberstein E, Zivin K (2009) Stigma and Help Seeking for Mental Health Among College Students. Medical Care Research and Review 66(5): 522-541.

15. Simon HA (1997) Administrative Behavior: A Study of Decision-Making Processes in Administrative Organization. $4^{\text {th }}$ (Edn.), The Free Press, New York.

16. Kessler RC, Berglund P, Demler O, Jin R, Merikangas $\mathrm{KR}$, et al. (2005) Lifetime prevalence and age-of-onset distributions of DSM-IV disorders in the National Comorbidity Survey Replication. Archives of General Psychiatry 62(6): 593-602. 